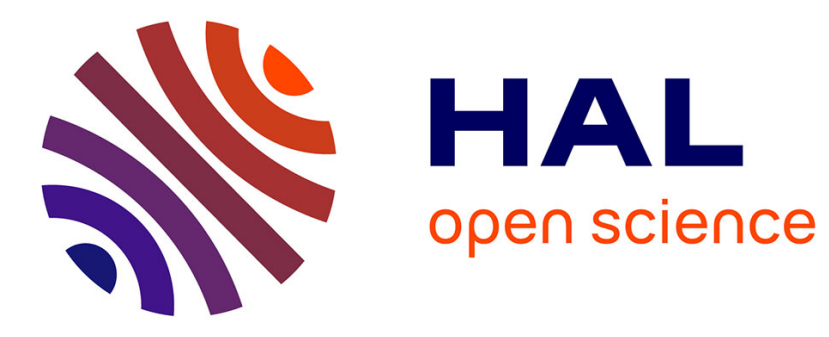

\title{
A Framework for Multi-angle Tirf Microscope Calibration
}

Emmanuel Soubies, Sébastien Schaub, Agata Radwanska, Ellen van Obberghen-Schilling, Laure Blanc-Féraud, Gilles Aubert

\section{To cite this version:}

Emmanuel Soubies, Sébastien Schaub, Agata Radwanska, Ellen van Obberghen-Schilling, Laure BlancFéraud, et al.. A Framework for Multi-angle Tirf Microscope Calibration. ISBI - International Symposium on Biomedical Imaging, Apr 2016, Prague, Czech Republic. pp.4, 10.1109/ISBI.2016.7493355 . hal-01257736

\section{HAL Id: hal-01257736 https://inria.hal.science/hal-01257736}

Submitted on 18 Jan 2016

HAL is a multi-disciplinary open access archive for the deposit and dissemination of scientific research documents, whether they are published or not. The documents may come from teaching and research institutions in France or abroad, or from public or private research centers.
L'archive ouverte pluridisciplinaire HAL, est destinée au dépôt et à la diffusion de documents scientifiques de niveau recherche, publiés ou non, émanant des établissements d'enseignement et de recherche français ou étrangers, des laboratoires publics ou privés. 


\title{
A FRAMEWORK FOR MULTI-ANGLE TIRF MICROSCOPE CALIBRATION
}

\author{
Emmanuel Soubies ${ }^{1 \star}$, Sébastien Schaub ${ }^{2 \star}$, Agata Radwanska ${ }^{2}$, \\ Ellen Van Obberghen-Schilling ${ }^{2}$, Laure Blanc-Féraud ${ }^{1 \star}$ and Gilles Aubert $^{3}$ \\ ${ }^{1}$ Université Nice Sophia Antipolis, I3S, CNRS, Sophia Antipolis, France. \\ ${ }^{2}$ Université Nice Sophia Antipolis, iBV, CNRS, INSERM, Nice, France. \\ ${ }^{3}$ Université Nice Sophia Antipolis, Laboratoire J.A. Dieudonné, CNRS, Nice, France.
}

\begin{abstract}
This communication presents a pipeline for Multi-Angle TIRF calibration from the measurement of the incident angle to the model validation. This problem is of major importance when dealing with 3D reconstruction methods from a set of MA-TIRF acquisitions since the reconstruction accuracy highly depends on the agreement between the theoretical model and the physical system. One main issue is then to build phantom samples with known geometry, or known properties, in order to adjust and/or validate the model. This paper describes such a calibration procedure using a lens as phantom sample and proposes a new model validation experiment based on a dual-color co-localization.
\end{abstract}

Index Terms - Multi-Angle TIRF microscopy - image reconstruction - fluorescence imaging - microscope calibration.

\section{INTRODUCTION}

Total Internal Reflection Fluorescence microscopy (TIRF) is a method adapted to visualize membrane-substrate interactions. The principle of this device relies on the total internal reflection phenomenon generating an evanescent wave capable of producing a selective excitation of the dye molecules within a single layer of 100 to 500nm [4]. The fast decay of the evanescent wave varies with respect to the incident angle of the light beam. Hence, intensity variations on TIRF images, occurring when changing the incident angle, are, in part, due to the axial positions of the observed structures. While a direct interpretation of Multi-Angle TIRF (MA-TIRF) images in terms of axial structure positions is not an easy task, reconstruction algorithms can be dedicated to compute a quantitative depth map with high axial resolution. Thus, MA-TIRF microscopy, combined with reconstruction algorithms, has been successfully used to visualize sub-cellular structures in the vicinity of the cell membrane $[1,2,3]$ (see also refs. therein). However, the success of such reconstruction methods strongly depends on the system calibration. One needs to ensure an accurate control of the incident angle governing the evanescent wave decay and validate the theoretical TIRF model with respect to the microscopy device. Such a validation requires the construction of phantom samples for which the geometry is known accurately. The following work takes place within this context.

Contributions and outline The goal of this paper is to present an alternative (i.e. using simple protocols) framework for MA-TIRF

* Authors belonging to the Morpheme team (INRIA/CNRS/UNS). Contact: lastname@unice.fr calibration. In Section 2, the MA-TIRF system as well as the mathematical modeling are described and the main calibration issues are outlined. Then, Section 3 and 4 present some methods of the state of the art, respectively dedicated to angle calibration and model validation, and adapt them to our device. In particular, we detail numerical methods to extract useful data from the acquired images and use them to calibrate the system. Finally, in Section 5, a new experiment based on dual-color co-localization is proposed to complete the MA-TIRF model validation.

\section{SYSTEM DESCRIPTION}

MA-TIRF setup Our MA-TIRF microscope has been designed to control and change rapidly the illumination angle on the sample. We conjugate galvanometric mirrors (fast and accurate flipping mirrors) and coupling optics to externally control the illumination angle (Fig. 1). Moreover, using 2axis galvanometric mirrors allows fast azimuthal rotation during image acquisition homogenizing the sample illumination and allowing to ignore local polarization properties of the sample (see [2] and refs. therein).

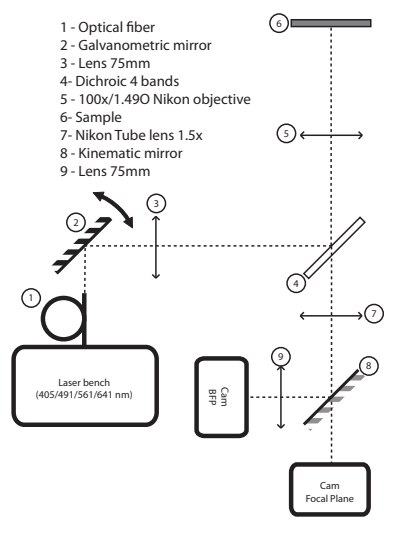

Fig. 1: TIRF system.

MA-TIRF modeling Let us consider a set of $L$ incident angles $\mathcal{A}=\left\{\alpha_{1}, \ldots, \alpha_{L}\right\}$ greater than the critical angle $\alpha_{c}$ (i.e. $\forall \alpha \in$ $\mathcal{A}, \alpha>\alpha_{c}$ ). Then, a MA-TIRF acquisition produces a set of 2D images, $\mathcal{S}:=\left\{\mathrm{s}(\cdot, \alpha): \Omega \subset \mathbb{R}^{2} \rightarrow \mathbb{R}_{+}, \alpha \in \mathcal{A}\right\}$, according to the following forward model: $\forall \mathrm{x} \in \Omega, \forall \alpha \in \mathcal{A}$,

$$
\mathrm{s}(\mathrm{x}, \alpha)=I_{0}(\alpha) \int_{0}^{\infty} f(\mathrm{x}, z) e^{-z p(\alpha)} \mathrm{d} z+b_{\mathrm{x}}
$$

where $^{1} f$ denotes the 3D fluorophore density, $b_{\mathrm{x}}$ a background constant (in $z$ ) signal at the position $\mathrm{x}$ and $p(\alpha)$ is the inverse of the penetration depth of the evanescent wave in the sample, depending on the excitation wavelength as well as both the incident (glass) and transmitted (sample) refractive indices [4]. Finally, $I_{0}$ models the

\footnotetext{
${ }^{1}$ Here the variable $\mathrm{x} \in \Omega$ denotes the couple $(x, y)$ defining a position in the image domain $\Omega \subset \mathbb{R}^{2}$.
} 
intensity at the interface (i.e. $z=0$ ) for which a theoretical expression, taking into account the azimuthal rotation of the laser beam, can be found in [2].

Then, from the set of 2D acquisitions $\mathcal{S}$, one aims to recover the 3D fluorophore density $f$, that is, solve the inverse problem. It is thus crucial to calibrate the system to be in accordance with model (1). Two main issues are concerned: i) how can we measure precisely the incident angle $\alpha$ ? ii) is the theoretical model (1) in agreement with the physical device? These questions are addressed in the next sections.

\section{INCIDENT ANGLE CALIBRATION}

Accurate 3D reconstruction from MA-TIRF acquisitions requires a precise control of the incident angle. According to [1], this can be achieved considering back focal plane (BFP) images of several solutions differing by their refractive indices. These experiments require to spin-coat quantum dots to create a 2D-fluorescent object. In the method we propose below, it only requires homogeneous fluorescent solutions of several refractive indices.

Theoretical relation tension/angle From the tension $U_{g v}$ applied to the galvanometric mirror controlling the laser beam orientation, one can obtain the incident angle $\alpha$ using the relation:

$$
\alpha=\arcsin \left(\frac{\sin \left(K U_{g v}\right)}{F_{o b j} n_{i}} F_{1}\right)
$$

where $F_{o b j}=2 \mathrm{~mm}$ and $F_{1}=75 \mathrm{~mm}$ are respectively the focal distances of the objective and the lens 3 in Fig. 1, $n_{i}=1.518$ denotes the refractive index of the incident medium (glass) and $K$ is a characteristic constant of the galvanometric mirror specified by the manufacturer to be $2^{\circ} \cdot \mathrm{V}^{-1}$. The goal of the following experiments is thus to validate this relation and adjust the value of $K$.

Protocol Considering a homogeneous fluorescent solution of refractive index $n_{t}$, we known from Descartes' laws that the associated critical angle is given by $\alpha_{c}=\arcsin \left(n_{t} / n_{i}\right)$. In MA-TIRF microscopy, when observing the BFP of the objective for such a solution, we can distinguish two bright spots corresponding respectively to the incident and the reflected beams. These spots are superimposed when $\alpha=0^{\circ}$ and move along a line, symmetrically to this center position, when the incident angle $\alpha$ increases. In our setup, due to the azimuthal rotation, we observe a ring with a radius increasing with $\alpha$ (Fig. 2).
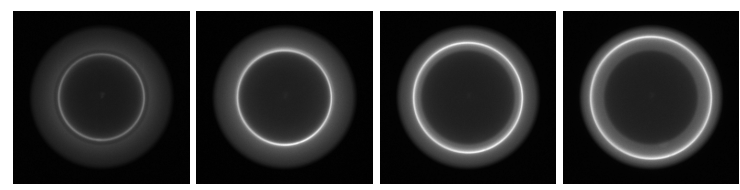

Fig. 2: Ring evolution on the BFP with respect to the incident angle $\alpha$ which increases from left to right (interface glass - air).

Due to the total reflection phenomenon, when $\alpha>\alpha_{c}$ the ring becomes brighter and computing the mean of the BFP images acquired for different angles $\alpha \in \mathcal{A}$ (Fig. 3) reveals the BFP ring position corresponding to $\alpha_{c}$ as well as the one associated to the maximal angle $\alpha_{\max }=\sin ^{-1}\left(\mathrm{NA} / n_{i}\right)$, where NA denotes the objective numerical aperture. The dashed blue circle in Fig. 3 corresponds to the first angle of the scanning used to compute the mean image. The circle corresponding to $\alpha_{c}$ can be easily detected using the Circle Hough Transform [5] (Matlab function imfindcircles). In this way, we obtain a relation between the angle $\alpha_{c}$ and the radius of the associated circle denoted $r_{c}$. Then, in order to get a correspondence

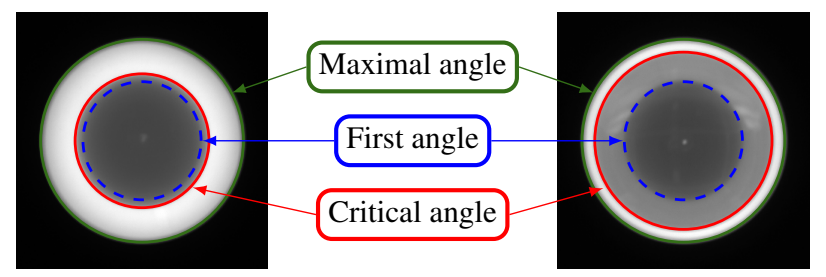

Fig. 3: Mean of the BFP images for two interfaces. Left: glass - air ( $\alpha_{c}=$ $\left.41.2^{\circ}\right)$. Right: glass - water $\left(\alpha_{c}=61.18^{\circ}\right)$.

between $\alpha_{c}$ and the "critical tension" $U_{g v}^{c}$ applied to the galvanometric mirror, one can determine, within the acquired BFP stack of images (Fig. 2), the frame on which the ring has a radius equal to $r_{c}$. This can be performed using simple numerical methods based on radial lines from the center of the circles detected in Fig. 3. Repeating this procedure with homogeneous solutions of different refractive indices $n_{t}$ leads to a set of couples $\left\{\left(\alpha_{c}, U_{g v}^{c}\right)\right\}$ from which the model (2) can be adjusted. Interfaces glass-air, glass-water and glass-"sucrose" 2 are considered in the latter.

Model fitting Fig. 4 presents the set of points $\left\{\left(\alpha_{c}, U_{g v}^{c}\right)\right\}$ obtained respectively in January and April $2015^{3}$. First of all, one can notice the proximity of the points of January and April showing the stability of the system. Then, we plot on the same graph the theoretical curve (i.e. model (2) for $K=2^{\circ} . \mathrm{V}^{-1}$ ) in dashed blue. Clearly, this model does not represent the measured data. However, computing a least-square fitting with respect to $K$ allows to significantly improve the agreement between model and data points (black curve in Fig 4). The resulting value for $K$ is then $1.95^{\circ} . \mathrm{V}^{-1}$.

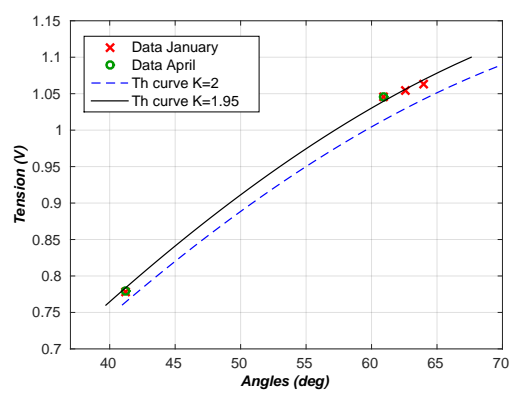

Fig. 4: Curve angle/tension according to the model (2) for $K=2$ (manufacturer specifications) in dashed blue and $K=1.95$ (fit w.r.t. $K$ ) in black.

\section{DECAY PROFILE VALIDATION}

The second important point discussed above concerns the validation of the theoretical TIRF model (1). The response of a biological specimen through the MA-TIRF acquisition system strongly depends on the decay of the evanescent wave, known to be exponential from

\footnotetext{
${ }^{2}$ We use solutions with different sucrose concentrations leading to indices greater than $n_{t}=1.333$ (water).

${ }^{3}$ April data were acquired only for interfaces glass-air and glass-water.
} 
TIRF theory [4]. Hence, the 3D reconstruction accuracy is directly related to the modeling of this decaying function. It is thus crucial to verify if the simple model (1) is sufficient to describe our setup. In order to do such a validation, one needs to use a sample of known geometry (i.e. a phantom sample). For instance, authors in [2] used small beads placed on an inclined plane with a known slope. Here, we propose to use a method similar to the one used in [3] and described in the next paragraph.

Protocol The procedure to build the phantom sample is very simple. A lens with a large radius of curvature $R_{c}$ and diameter $\varnothing$ (here $R_{c}=288.2 \mathrm{~mm}$ and $\varnothing=25.4 \mathrm{~mm}$ ) is placed into a homogeneous fluorescent solution as showed in Fig. 5. Then, MA-TIRF images are acquired in a zone containing the border of the lens (in red in Fig. 5). Since the dimension of the lens is huge with respect to the size of the observed region, the depth of the homogeneous solution below the lens can be assumed to increases linearly from the border to the center of the lens. One can also imagine to replace the lens by a large bead as it was done in [3]. However, when using such a bead, acquired images can suffer from reflection phenomenon due to the particular geometry of the bead. Note that the acquired images of the phantom using the lens can also be used to estimate the decay function in the same spirit as in [6].

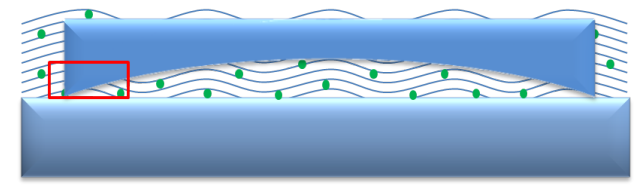

Fig. 5: Phantom sample constructed from a large lens and an homogeneous fluorescent solution. The red rectangle represents the observed region through the MA-TIRF setup.

Reconstruction method Since we know that the observed sample has a particular geometry, we will use a strong shape prior for the reconstruction. For each pixel $\mathrm{x} \in \Omega$, we consider that the uniform fluorescent layer $f$ defines a Top-Hat function with respect to $z$,

$$
\forall(\mathrm{x}, z) \in \Omega \times\left[0,+\infty\left[, f(\mathrm{x}, z)=C_{\mathrm{x}} \mathbb{1}_{\left\{z \leq \bar{Z}_{\mathrm{x}}\right\}},\right.\right.
$$

where $C_{\mathrm{x}} \in \mathbb{R}_{+}$and $\bar{Z}_{\mathrm{x}} \in \mathbb{R}_{+}$denote respectively the fluorophore "concentration" and the depth of the homogeneous solution at the position $\mathrm{x}$. This form was previously used in [3] and one can obtain an explicit expression for the integral defining the TIRF model (1), $\forall \mathrm{x} \in \Omega, \forall \alpha \in \mathcal{A}$,

$$
\mathrm{s}(\mathrm{x}, \alpha)=\frac{C_{\mathrm{x}}}{p(\alpha)} I_{0}(\alpha)\left[1-e^{-\bar{Z}_{\mathrm{x}} p(\alpha)}\right]+b_{\mathrm{x}} .
$$

Then, for each $\mathrm{x} \in \Omega$, this model can be fitted to the measurements to get an estimation of the variables $C_{\mathrm{x}}, \bar{Z}_{\mathrm{x}}$ and $b_{\mathrm{x}}$. An acquired image (for $\alpha \in \mathcal{A}$ ) is presented in Fig. 6 (bottom) for illustration. Note that it is crucial to consider the background $b_{\mathrm{x}}$ when imposing a positivity constraint on $f$ as outlined in [2] where the authors use an extra dark image to estimate this background and remove it from all the acquired images. Here, we consider the background as a variable and estimate it conjointly with the fluorophore density $f$ while imposing the positivity of these unknowns. Finally, the nonlinear least-squares fit with positivity constraint is performed using the Levemberg-Marquardt algorithm [7].
Results Fitted curves are presented in Fig. 6 (Top) for different positions $\mathrm{x} \in \Omega$. We can see that the model is in good agreement with the data which means that there are parameters $C_{\mathrm{x}}, \bar{Z}_{\mathrm{x}}$ and $b_{\mathrm{x}}$ such that model (4) is able to represent the measured data accurately. The remaining question concerns the values of the estimated parameters: are they coherent with the characteristics of the lens?

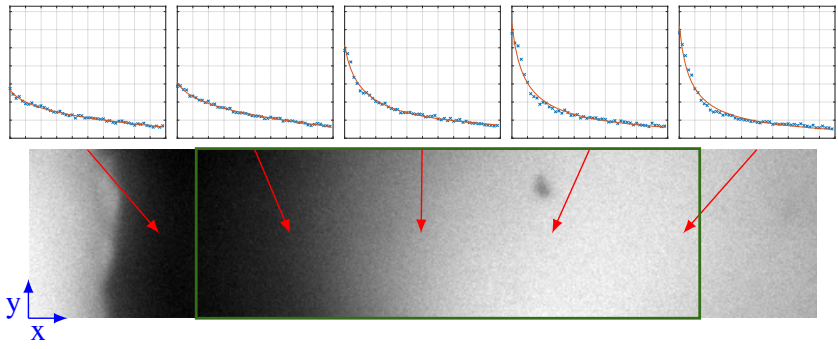

Fig. 6: Top: Fit of (4) to the acquired data at different positions $\mathrm{x} \in \Omega$. Blue crosses correspond to the data and the fitted curve is in red (Angles Vs Intensity). Bottom: an acquired image of the phantom (Fig 5) for $\alpha \in \mathcal{A}$.

In order to answer this question, we compute the mean value of $\bar{Z}_{\mathrm{x}}, \mathrm{x} \in \Omega$ along the $\mathrm{Y}$ direction as well as the corresponding standard deviation within the green zone of Fig. 6. Results are presented in Fig. 7 where a line has been fitted using the points between the two dashed lines. We have restricted the fit to these points since the accuracy of the reconstruction decreases when $\bar{Z}_{\mathrm{x}}$ increases and that we are not ensured that the profile of the lens is still linear for points close to the lens border. Indeed, this border is not infinitesimally thin and is sanded down to ensure safety during manipulations.

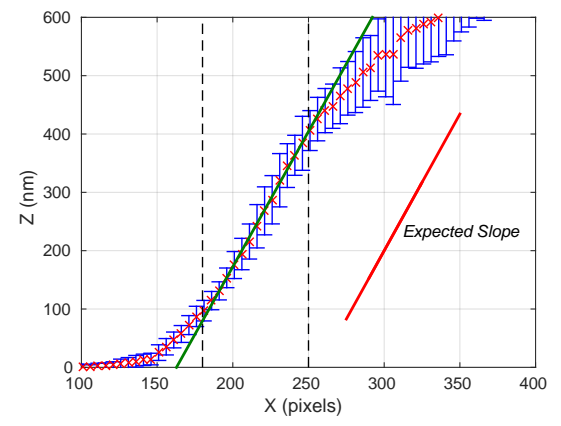

Fig. 7: Mean and standard deviation (along the $\mathrm{Y}$ direction) of estimated $\bar{Z}_{\mathrm{x}}$ positions corresponding to the green zone of Fig. 6 . The green line has been fitted using data points between the two dashed lines. Expected slope is represented in red.

Since the characteristics $R_{c}$ and $\varnothing$ of the lens are known, one can determine the expected slope to be $2.53^{\circ}$ (using the fact that 1 pixel $=106 \mathrm{~nm}$ for $X$ ) which is in accordance with the fitted green line in Fig. 7 whose slope is equal to $2.51^{\circ}$. One can notice that the estimated $\bar{Z}_{\mathrm{x}}$ accurately follow the expected slope of the lens until a limit around $400 \mathrm{~nm}$ while in previous experiments performed on phantom samples $[2,3]$, results where displayed only over the first $200 \mathrm{~nm}$. This shows a limitation to accurately reconstruct deep structures as expected with this microscopy device. Finally, we observe a loss of precision at the vicinity of the glass coverslip which can be due, as indicated previously, to the irregularities of the lens border as well as the collection efficiency, not modeled in (1), which is disturbed by the glass surface for proximal fluorophores [6, and refs. therein]. 


\section{DUAL-COLOR CO-LOCALIZATION}

In this section, we present a new experiment to further complete the model validation procedure. Since our system is able to perform multiwavelength acquisitions, and the model depends on the excitation wavelength, the idea is to use a sample for which the structures of interest are labeled using two different fluorescent proteins sensitive to different wavelengths and emitting respectively green and red fluorescence. Thus, we obtain two independent acquisitions for which reconstruction results are expected to co-localize.

Materiel and methods Human umbilical vein endothelial cells were stained with conformation-dependent monoclonal anti- $\alpha 5 \beta 1$ integrin antibodies and incubated with both Alexa Fluor488 (green) and Alexa Fluor546 (red) conjugated secondary anti-mouse antibodies. MA-TIRF acquisitions were performed using 50 incident angles.

Reconstruction method Assuming that data are corrupted by a Gaussian noise and imposing the fluorophore density and the background to be positive, we are concerned by solving the following optimization problem,

$$
\{\hat{\mathrm{f}}, \hat{b}\} \in \arg \min _{\mathrm{f}, b} \frac{1}{2}\|A(\mathrm{f}, b)-\mathrm{s}\|_{2}^{2}+\mathcal{X}_{\geq 0}([\mathrm{f}, b]),
$$

where $A, \mathrm{f}$ and $b$ are discrete versions of, respectively, the TIRF operator (1), the fluorophore density (3D image) and the background image (2D), and, $\mathcal{X}_{\geq 0}(x)=\{0$ if $x \geq 0,+\infty$ otherwise $\}$ denotes the characteristic function of positive vectors. Note that there is no spatial regularization in (5), such as the well-known TV regularizer used in [2]. Indeed, we did not want to impose too much prior on the solution since we observed on simulated 1D examples (not presented here due to page limitation) that TV regularization can affect estimation of deeper objects. Moreover, reconstructions presented in the following are minimally affected by the noise. Finally, problem (5) is solved through the Chambolle-Pock algorithm [8].

Results Reconstructions were preformed with an axial discretization of $10 \mathrm{~nm}$ steps and considering a wavelength-dependent index $n_{t}$ of the sample (eq. to water), different for Alexa488 $(\simeq 1.34$ ) and Alexa546 ( $\simeq 1.335)$ according to [9]. Results are presented in Fig. 8 using a color-coded depth representation to visualize such thin $3 \mathrm{D}$ volumes. Three extracted profiles are presented in Fig. 9 and localized by the arrows on the whole image (Fig. 8). One can appreciate on these profiles the co-localization between the two reconstructions obtained from two independent acquisitions. Moreover, we have to keep in mind that model (1) depends on the excitation wavelength which is different between the two acquisitions of this experiment. Thus, reconstruction are performed using two "versions" of model (1) and the good agreement between both reconstructions confirms the validity of this model to describe our microscopy system. Finally, results show a co-localization with a precision around $30-40 \mathrm{~nm}$ (which is the observed thickness of our fibers) over at least $170 \mathrm{~nm}$ depth.

\section{CONCLUSION}

In this paper we have proposed a framework for Multi-Angle TIRF calibration which is a crucial step in order to perform accurate $3 \mathrm{D}$ reconstructions of the observed sample. After calibrating the incident angle, we showed that model (1) is well suited to describe our physical device allowing to perform an accurate estimation of a phantom
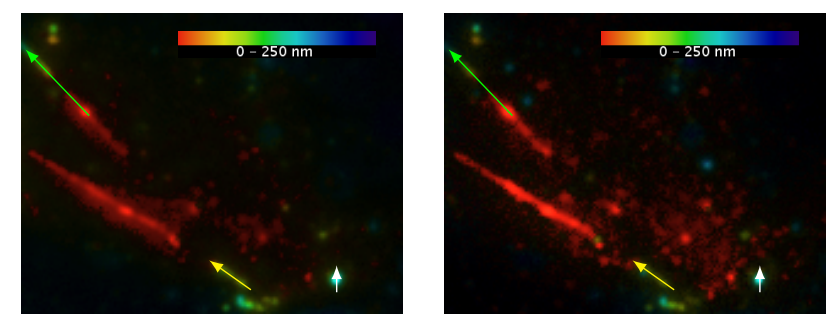

Fig. 8: Reconstruction results from the Alexa488 (left) and Alexa546 (right) acquisitions. Colors represent the depth of the biological structures. Arrows specify the location of the Fig. 9 extracted profiles.
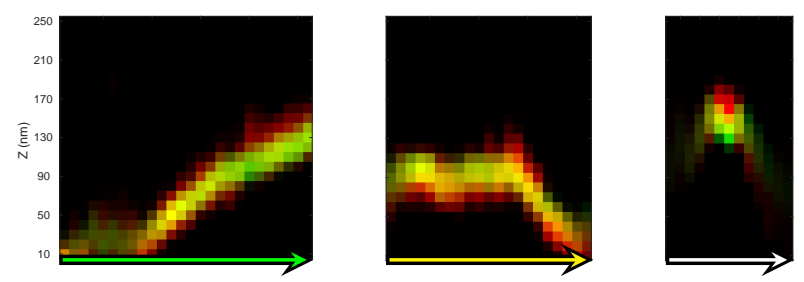

Fig. 9: Superimposition of Alexa488 (green) and Alexa546 (red) reconstructions along the green (left), yellow (center) and white (right) arrows of Fig. 8.

sample until 400nm depth. Then, the model validation is completed by a co-localization experiment which demonstrated the coherence between reconstructions obtained from two independent acquisitions using different excitation wavelength.

\section{REFERENCES}

[1] M. C. Dos Santos, R. Déturche, C. Vézy, and R. Jaffiol, "Axial nanoscale localization by normalized total internal reflection fluorescence microscopy," Optics letters, vol. 39, no. 4, 2014.

[2] J. Boulanger, C. Gueudry, D. Münch, B. Cinquin, P. Paul-Gilloteaux, S. Bardin, C. Guérin, F. Senger, L. Blanchoin, and J. Salamero, "Fast high-resolution $3 \mathrm{~d}$ total internal reflection fluorescence microscopy by incidence angle scanning and azimuthal averaging," Proceedings of the National Academy of Sciences, vol. 111, no. 48, 2014.

[3] B. P. Ölveczky, N. Periasamy, and A. S. Verkman, "Mapping fluorophore distributions in three dimensions by quantitative multiple angletotal internal reflection fluorescence microscopy.," Biophysical journal, vol. 73, no. 5, 1997.

[4] D. Axelrod, E. H. Hellen, and R. M. Fulbright, "Total internal reflection fluorescence," in Topics in fluorescence spectrometry. Springer, 2002.

[5] R.O. Duda and P.E. Hart, "Use of the hough transformation to detect lines and curves in pictures," Com. ACM, vol. 15, no. 1, 1972.

[6] A. L. Mattheyses and D. Axelrod, "Direct measurement of the evanescent field profile produced by objective-based total internal reflection fluorescence," J. of biomedical optics, vol. 11, no. 1, 2006.

[7] C. Kanzow, N. Yamashita, and M. Fukushima, "Levenberg-marquardt methods with strong local convergence properties for solving nonlinear equations with convex constraints," J. of Comput. and App. Math., vol. 172, no. 2, 2004.

[8] A. Chambolle and T. Pock, "A first-order primal-dual algorithm for convex problems with applications to imaging," J. of Math. Imaging and Vision, vol. 40, no. 1, 2011.

[9] David J Segelstein, The complex refractive index of water, Ph.D. thesis, University of Missouri-Kansas City, 2011. 